\author{
Marquette University \\ e-Publications@Marquette
}

Biomedical Engineering Faculty Research and

Publications

Biomedical Engineering, Department of

$11-2017$

\title{
Sagittal Subtalar and Talocrural Joint Assessment During Ambulation With Controlled Ankle Movement (CAM) Boots
}

\author{
Ben McHenry \\ Marquette University, ben.mchenry@marquette.edu \\ Emily L. Exten \\ Medical College of Wisconsin \\ Janelle A. Cross \\ Medical College of Wisconsin \\ Karen M. Kruger \\ Marquette University, karen.kruger@marquette.edu \\ Brian Law \\ Medical College of Wisconsin
}

See next page for additional authors

Follow this and additional works at: https://epublications.marquette.edu/bioengin_fac

Part of the Biomedical Engineering and Bioengineering Commons

\section{Recommended Citation}

McHenry, Ben; Exten, Emily L.; Cross, Janelle A.; Kruger, Karen M.; Law, Brian; Fritz, Jessica M.; and Harris, Gerald F., "Sagittal Subtalar and Talocrural Joint Assessment During Ambulation With Controlled Ankle Movement (CAM) Boots" (2017). Biomedical Engineering Faculty Research and Publications. 541.

https://epublications.marquette.edu/bioengin_fac/541 


\section{Authors}

Ben McHenry, Emily L. Exten, Janelle A. Cross, Karen M. Kruger, Brian Law, Jessica M. Fritz, and Gerald F. Harris 
Marquette University

e-Publications@Marquette

\section{Biomedical Engineering Faculty Research and Publications/College of} Engineering

This paper is NOT THE PUBLISHED VERSION; but the author's final, peer-reviewed manuscript. The published version may be accessed by following the link in th citation below.

Foot and Ankle International, Vol. 38, No. 11 (November 1, 2017): 1260-1266. DOI. This article is (C) SAGE Publications and permission has been granted for this version to appear in $\mathrm{e}-$

Publications@Marquette. SAGE Publications does not grant permission for this article to be further copied/distributed or hosted elsewhere without the express permission from SAGE Publications.

\section{Sagittal Subtalar and Talocrural Joint Assessment During Ambulation with Controlled Ankle Movement (CAM) Boots}

Benjamin D. McHenry

Department of Biomedical Engineering, Marquette University, Milwaukee, WI Emily L. Exten Department of Orthopaedic Surgery, OhioHealth Mansfield Campus, Division of Foot and Ankle Surgery, Mansfield, $\mathrm{OH}$ Janelle A. Cross

Department of Orthopaedic Surgery, Medical College of Wisconsin, Milwaukee, WI Karen M. Kruger

Department of Biomedical Engineering, Marquette University, Milwaukee, WI

Brian Law

Department of Orthopaedic Surgery, Division of Foot and Ankle Surgery, Medical College of Wisconsin, Milwaukee, WI 


\section{Jessica M. Fritz}

Department of Biomedical Engineering, Marquette University/The Medical College of Wisconsin, Milwaukee, WI

\section{Gerald Harris}

Department of Biomedical Engineering, Marquette University/The Medical College of Wisconsin, Milwaukee, WI

\section{Abstract \\ Background:}

The purpose of the current study was to determine sagittal plane talocrural and subtalar kinematic differences between barefoot and controlled ankle movement (CAM) boot walking. This study used fluoroscopic images to determine talar motion relative to tibia and calcaneal motion relative to talus.

\section{Methods:}

Fourteen male subjects (mean age $24.1 \pm 3.5$ years) screened for normal gait were tested. A fluoroscopy unit was used to collect images at $200 \mathrm{~Hz}$ during stance. Sagittal motion of the talocrural and subtalar joints were analyzed barefoot and within short and tall CAM boots.

\section{Results:}

Barefoot talocrural mean maximum plantar and dorsiflexion were $9.2 \pm 5.4$ degrees and $-7.5 \pm 7.4$ degrees, respectively; short CAM boot mean maximum plantar and dorsiflexion were $3.2 \pm 4.0$ degrees and $-4.8 \pm 10.2$ degrees, respectively; and tall CAM boot mean maximum plantar and dorsiflexion were $-0.2 \pm 3.5$ degrees and $-2.4 \pm 5.1$ degrees, respectively. Talocrural mean range of motion (ROM) decreased from barefoot $(16.7 \pm 5.1$ degrees) to short CAM boot ( $8.0 \pm 4.9$ degrees) to tall CAM boot $(2.2 \pm 2.5$ degrees). Subtalar mean maximum plantarflexion angles were $5.3 \pm 5.6$ degrees for barefoot walking, $4.1 \pm 5.9$ degrees for short CAM boot walking, and $3.0 \pm 4.7$ degrees for tall CAM boot walking. Mean minimum subtalar plantarflexion angles were $0.7 \pm 3.2$ degrees for barefoot walking, $0.7 \pm 2.9$ degrees for short CAM boot walking, and $0.1 \pm 4.8$ degrees for tall CAM boot walking. Subtalar mean ROM decreased from barefoot ( $4.6 \pm 3.9$ degrees) to short CAM boot $(3.4 \pm 3.8$ degrees) to tall CAM boot $(2.9 \pm 2.6$ degrees $)$.

\section{Conclusion:}

Tall and short CAM boot intervention was shown to limit both talocrural and subtalar motion in the sagittal plane during ambulation. The greatest reductions were seen with the tall CAM boot, which limited talocrural motion by $86.8 \%$ and subtalar motion by $37.0 \%$ compared to barefoot. Short CAM boot intervention reduced talocrural motion by $52.1 \%$ and subtalar motion by $26.1 \%$ compared to barefoot.

\section{Clinical Relevance:}

Both short and tall CAM boots reduced talocrural and subtalar motion during gait. The short CAM boot was more convenient to use, whereas the tall CAM boot more effectively reduced motion. In treatments requiring greater immobilization of the talocrural and subtalar joints, the tall CAM boot should be considered.

Keywords gait analysis, hindfoot, subtalar joint, talocrural joint, ankle joint, biomechanics, weightbearing, fluoroscopy, sagittal motion 


\section{Introduction}

Orthotic walking boots, such as fracture boots, or controlled ankle movement (CAM) boots, are meant to immobilize the ankle joint complex in both nonoperative and postoperative treatment of acute and chronic injuries. $2,13,14,17$ These boots are often favorable to traditional cast immobilization techniques because of their lower cost, $, \underline{6}, \underline{13}, 15, \underline{17}$ ability to be removed for therapeutic exercises/wound inspection, $,-6,13,15, \underline{17}$ and ease of adjustability during prolonged treatment periods.., 15 This increased use of orthotic walking boots has led to reports in the literature about their effect on ground reaction force characteristics,,$\frac{6,17}{\text { plantar pressures, },, 11}$ and lower extremity kinematics. $-\underline{5}, \underline{13}-\underline{15}, \underline{17}$

Of the studies focused on lower extremity kinematics, only 3 tested ankle joint motion. $, \underline{14}, \underline{17}$ In 2001 , Raikin et al evaluated the ability of different braces to resist ankle/hindfoot sagittal and coronal torque when applied to a prosthetic ankle-foot complex within a test frame..$^{14}$ Authors concluded that the fracture boot provided less resistance to dorsiflexion, plantarflexion, eversion, and inversion when compared to cast immobilization. However, their study did not simulate walking or test a barefoot condition, and it is unclear if the prosthetic results would be replicated by an in vivo foot.

In 2008, Kadakia et al used radiographs to determine maximum plantar and dorsiflexion angles in 4 different types of walking boots compared to fiberglass cast treatment. ${ }^{5}$ The authors concluded that sagittal plane motion is restricted more with a fiberglass cast compared to any of the 4 types of walking boots. Measurements for their study were taken from radiographs of static foot positions (maximum plantar and dorsiflexion), and they only measured the total tibiopedal angle (angle between a line drawn along the posterior border of the tibia and a metal plate under the foot). The individual contributions of talocrural and subtalar sagittal plane motion were not analyzed and it is unknown if these results would be replicated during walking conditions.

Additionally, in 2006, Zhang et al reported no major changes to peak ankle dorsiflexion or sagittal plane range of motion (ROM) when comparing walking boot trials to shod trials, but saw a significant reduction in ankle eversion and frontal plane ROM..$^{17}$ Although the methodology of the study by Zhang et al describes foot marker placement (medial/lateral malleoli, head of first and fifth metatarsal), little information is given about segment definitions and kinematic validation. In addition, the study did not use radiographic imaging to determine foot position within the shoes or walking boots.

Orthotic bracing is ideally intended to control foot position while maintaining the ability to ambulate. ${ }^{14}$ It is therefore important to test their efficacy during ambulation when the dynamics of weightbearing, coupling segmental lower extremity motion, and synchronized muscle activity are present. This dynamic scenario differs significantly from simple static tests of ROM which do not assess the functional activity itself. Static ROM tests typically exceed those necessary for normal ambulation. ROM studies such as that of Raikin et al and Kadakia et al are of interest clinically, though the data were not collected during the actual functional activity (walking). ${ }^{5,14}$ Although the Zhang et al study did collect data with subjects walking, the lack of radiographic imaging prevented direct visualization of the bony position of the foot.

The purpose of the current study was to determine sagittal plane talocrural and subtalar kinematic differences between barefoot and CAM boot walking. This study used dynamic fluoroscopic imaging to determine talar motion relative to the tibia (talocrural) and calcaneal motion relative to the talus (subtalar) during ambulation. The kinematic model used in this study applied a combination of motion and fluoroscopic data and has previously been used to describe barefoot sagittal plane talocrural and subtalar motion while walking..$\underline{9} \underline{9}$ This fluoroscopic system has been evaluated and barefoot results have been compared to other studies using implanted bone pins. $1,7, \underline{9}$ 


\section{Materials and Methods}

In this institutional review board (IRB)-approved study, 14 male subjects (mean age $24.1 \pm 3.5$ years) screened for exclusion criteria gave written informed consent prior to being tested walking barefoot, in a short CAM boot (Short Walking Brace, Ovation Medical, Agoura Hills, CA), and in a tall CAM boot (Standard Walking Brace, Ovation Medical, Agoura Hills, CA) (Figure 1). Exclusion criteria included any significant injury to the foot and/or ankle or any previous lower extremity surgery (bilateral).

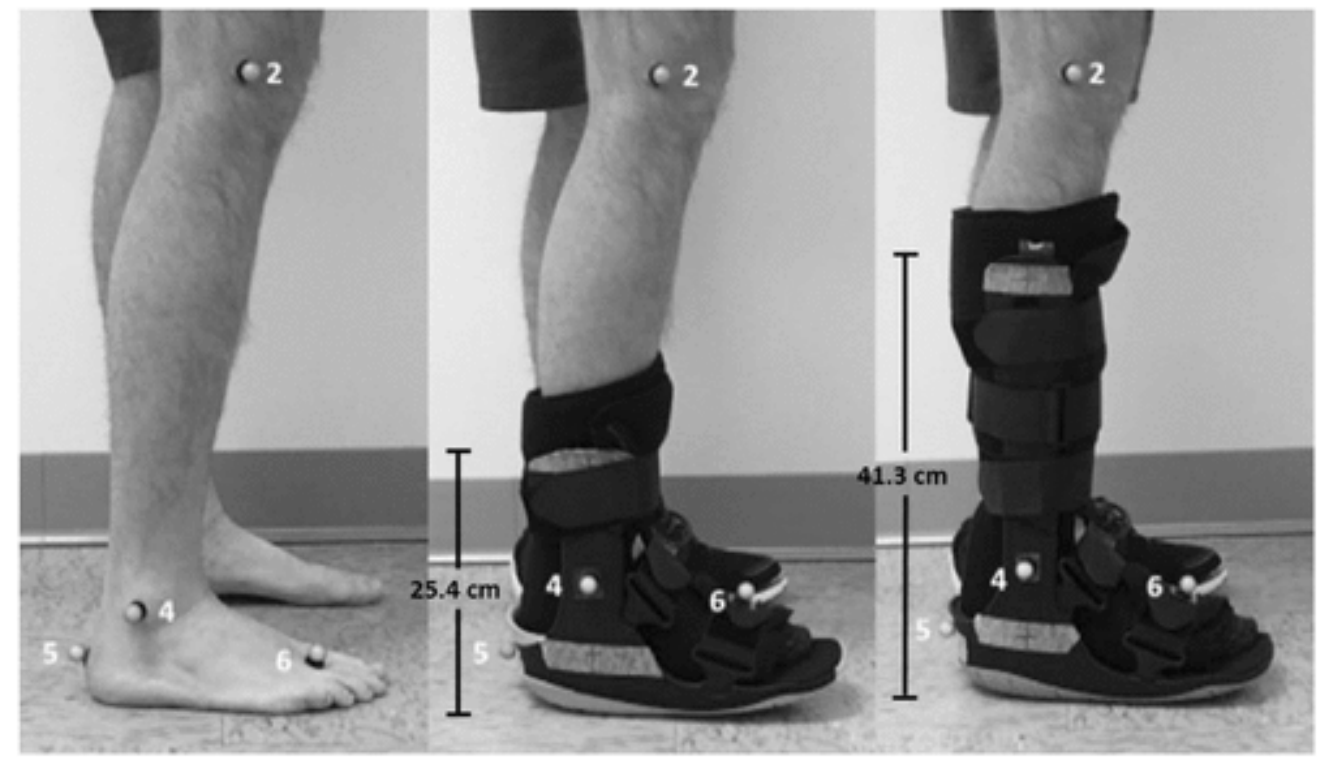

Figure 1. Barefoot (left), 12-in. short CAM boot (middle), and 18-in. tall CAM boot (right) walking conditions. Tibial contact location is measured from the floor to the most proximal point on the tibia constrained by each CAM boot. External markers 2, 4, 5, and 6 from Table 1 are also shown.

The data capture system consisted of a modified fluoroscopy unit $\frac{8}{-}$ placed within an existing Vicon MX motion analysis system (Vicon Motion Systems, Inc, Oxford, United Kingdom). The fluoroscopy unit (OEC 9000, GE, Fairfield, CT) was modified so that the image intensifier and emitter could be set on opposite sides of the walkway (Figure 2). Heel-strike and \pm -off events were detected using an embedded multiaxis force plate (AMTI, Watertown, MA).

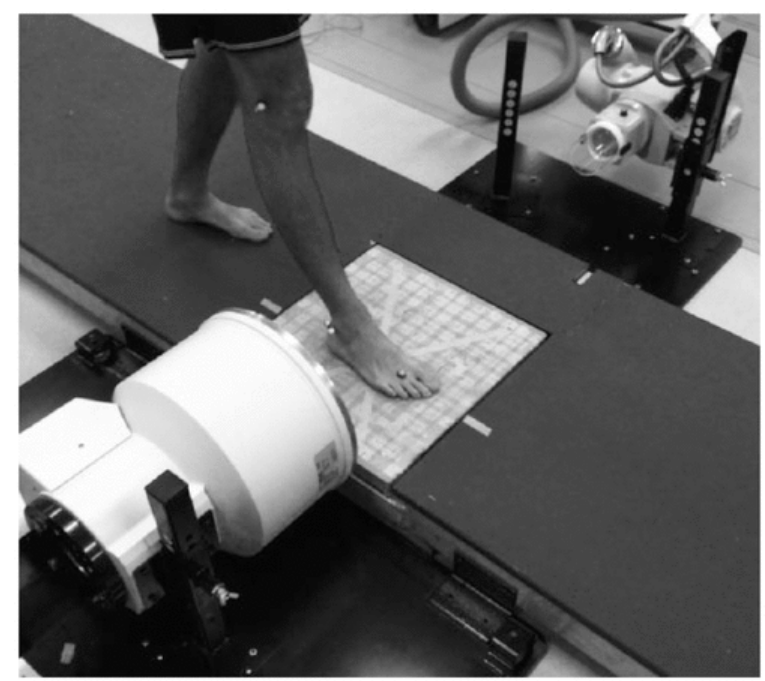

Figure 2. System configuration showing the walkway, emitter (farside), and image intensifier (nearside). Also shown is typical foot placement during image collection. Reprinted from McHenry et al. $\underline{9}$ 
The right leg and foot of each subject were instrumented with 6 reflective markers $(\mathrm{d}=16 \mathrm{~mm})$ in accordance with Table 1. For both the short and tall CAM boot trials, foot markers (medial/lateral malleoli, calcaneal tuberosity, and the head of second metatarsal) were placed directly on the CAM boot as close to its corresponding anatomic location as possible. Fluoroscopic and motion data were collected simultaneously (200 $\mathrm{Hz}$ ) as subjects walked along a custom walkway. Each subject completed 5 trials in each of the 3 conditions of walking barefoot, walking while wearing a short CAM boot, and walking while wearing a tall CAM boot. For the short and tall CAM boot trials, subjects wore an athletic sneaker on the contralateral foot. The effects of contralateral footwear were not investigated.

Table 1. External Marker Locations.

\begin{tabular}{|l|l|}
\hline Marker Number & Marker Location \\
\hline 1 & Medial femoral epicondyle \\
\hline 2 & Lateral femoral epicondyle \\
\hline 3 & Medial malleoli \\
\hline 4 & Lateral Malleoli \\
\hline 5 & Calcaneal tuberosity \\
\hline 6 & Second metatarsal head \\
\hline
\end{tabular}

Table I. External Marker Locations.

\begin{tabular}{ll}
\hline Marker Number & \multicolumn{1}{c}{ Marker Location } \\
\hline 1 & Medial femoral epicondyle \\
2 & Lateral femoral epicondyle \\
3 & Medial malleoli \\
4 & Lateral malleoli \\
5 & Calcaneal tuberosity \\
6 & Second metatarsal head \\
\hline
\end{tabular}

Radiation restrictions obviated recollection of fluoroscopic data if there was improper foot placement in the capture volume. Because of this, not all 14 subjects had 5 trials of acceptable data for each condition. For the barefoot condition, subjects averaged $4.6 \pm 0.6$ trials; for the short CAM boot condition, subjects averaged $4.3 \pm$ 0.9 trials; for the tall CAM boot condition, subjects averaged $4.8 \pm 0.6$ trials ( $n=191$ dynamic trials). Subject foot placement also determined how much of the stance phase could be analyzed for each trial. If the tibia vacated the fluoroscopic field of view during toe-off, the analysis stopped. Of the 191 dynamic trials analyzed, $89.0 \%$ of them spanned at least $90 \%$ of stance, $49.7 \%$ of them spanned at least $95 \%$ of stance, and $18.3 \%$ spanned the entire stance phase. In addition to the dynamic trials, static trials were collected for each subject in all 3 conditions (barefoot, short CAM boot, tall CAM boot) while standing in a natural weightbearing position ( $n=42$ static trials).

The kinematic model used in this study has been previously applied to describe barefoot talocrural and subtalar sagittal plane motion. ${ }^{8,9}$ The model used external marker position to define a tibial local coordinate system, and fluoroscopic markers to define talar and calcaneal local coordinate systems. External markers (medial/lateral malleoli and medial/lateral femoral epicondyles) were used to define the tibial local coordinate system as only the very distal end of the tibia was fluoroscopically visible for much of stance. For the talus and calcaneus, 2 points of interest per bone (talus, calcaneus) were translated from pixel coordinates to motion analysis global coordinates using a method of global referencing. This method has previously been shown to have errors less than $2 \mathrm{~mm}$ with subject foot progression angles of \pm 5 degrees. ${ }^{8}$ Average foot progression angle for the current study was -1.7 degrees ( $n=233,191$ dynamic trials and 42 static). These translated points of interest were 
defined in the sagittal plane of the foot and were then used to describe local coordinate systems for the talus and calcaneus. These local coordinate systems were then used to calculate talocrural and subtalar sagittal plane kinematics, with motion defined as distal position relative to proximal. Kinematics were also calculated from the statically collected data, and these angles served as neutral position. Kinematic repeatability using this system has been determined to be 1.06 degrees. ${ }^{8}$ Figure 3 illustrates the process of fluoroscopically assessing hindfoot motion as described in our previous work.

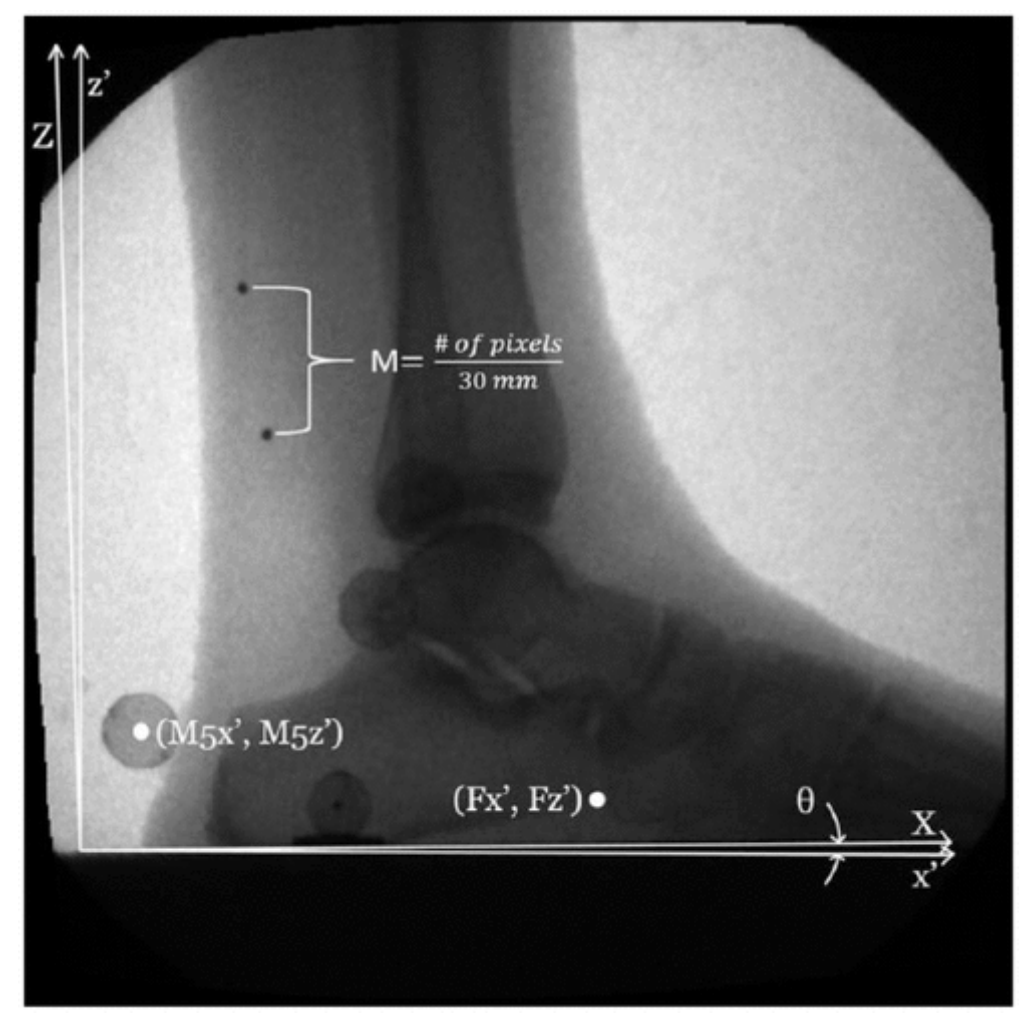

Figure 3. Fluoroscopic image showing a point of interest location that is translated from image coordinates (Fx', $\left.F z^{\prime}\right)$ to global coordinates using an external marker image ( $\left.M 5 x^{\prime}, M 5 z^{\prime}\right)$ and global coordinate locations, as well as the image magnification factor (M), subject foot progression angle (calculated from external marker M5 and M6 global coordinates), and camera static angular rotation from global $(\theta)$.

A 1-way analysis of variance (ANOVA) test was used to determine if there was a significant difference $(P \leq .05)$ in ROM of either joint (talocrural or subtalar) among the 3 conditions (barefoot, short CAM boot, tall CAM boot). If a significant result was determined at either joint using ANOVA test, then a least significant difference test was used to determine which direct comparison(s) was significantly different (barefoot vs short CAM boot, barefoot vs tall CAM boot, or short CAM boot vs tall CAM boot).

\section{Results}

All trials were averaged for assessment of each tested condition with barefoot comprising 64 trials, short CAM boot comprising 60 trials, and tall CAM boot comprising 67 trials. Talocrural kinematics for all 3 conditions are shown in Figure 4. Barefoot mean maximum plantar and dorsiflexion were $9.2 \pm 5.4$ degrees and $-7.5 \pm 7.4$ degrees, respectively, occurring at $9 \%$ and $82 \%$ stance phase. Short CAM boot mean maximum plantar and dorsiflexion were $3.2 \pm 4.0$ degrees and $-4.8 \pm 10.2$, respectively, occurring at $11 \%$ and $100 \%$ stance phase. Tall CAM boot mean maximum plantar and dorsiflexion were $-0.2 \pm 3.5$ degrees and $-2.4 \pm 5.1$ degrees, respectively, occurring at $17 \%$ and $94 \%$ stance phase. Talocrural mean ROM decreased from barefoot to short CAM boot to tall CAM boot from $16.7 \pm 5.1$ degrees to $8.0 \pm 4.9$ degrees to $2.2 \pm 2.5$ degrees (Table 2). There were significant 
differences between all talocrural mean ROM measurements (barefoot vs short CAM boot, barefoot vs tall CAM boot, and short CAM boot vs tall CAM boot).
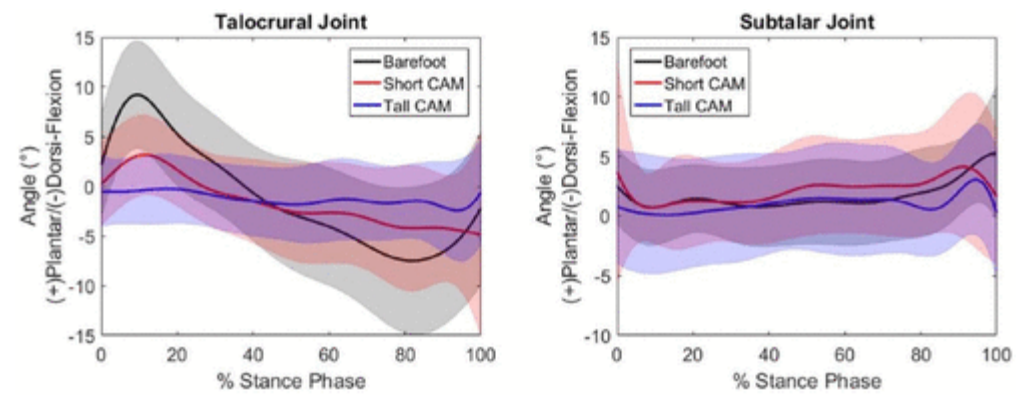

Figure 4. Talocrural (left) and subtalar (right) plantar/dorsiflexion angles during stance. The black solid line and gray band represents the mean and standard deviation of all 14 subjects walking barefoot, respectively. The red solid line and light red band represents the mean and standard deviation of all 14 subjects walking in the short CAM boot, respectively. The blue solid line and light blue band represents the mean and standard deviation of all 14 subjects walking in the tall CAM boot, respectively.

Table 2. Talocrural Kinematics During Stance Phase.

\begin{tabular}{|l|l|l|l|c|l|}
\hline & $\begin{array}{l}\text { Barefoot, } \\
\text { degrees }\end{array}$ & & $\begin{array}{l}\text { Short CAM Boot, } \\
\text { degrees }\end{array}$ & $\begin{array}{l}\text { Tall CAMBoot, } \\
\text { degrees }\end{array}$ \\
\hline & Mean (SD) & & Mean (SD) & & Mean (SD) \\
\hline Maximum plantarflexion & $9.2(5.4)$ & & $3.2(4.0)$ & & $-0.2(3.5)$ \\
\hline Maximum dorsiflexion & $-7.5(7.4)$ & & $-4.8(10.2)$ & & $-2.4(5.1)$ \\
Range of motion & $16.7(5.1)$ & $8.0(4.9)^{*}$ & $2.2(2.5)^{*}, t$ \\
\hline
\end{tabular}

*Significant difference compared to barefoot.

+Significant difference compared to short CAM boot.

Table 2. Talocrural Kinematics During Stance Phase.

\begin{tabular}{|c|c|c|c|}
\hline & Barefoot, degrees & Short CAM Boot, degrees & Tall CAM Boot, degrees \\
\hline & Mean (SD) & Mean (SD) & Mean (SD) \\
\hline Maximum plantarflexion & $9.2(5.4)$ & $3.2(4.0)$ & $-0.2(3.5)$ \\
\hline Maximum dorsiflexion & $-7.5(7.4)$ & $-4.8(10.2)$ & $-2.4(5.1)$ \\
\hline Range of motion & $16.7(5.1)$ & $8.0(4.9)^{2}$ & $2.2(2.5)^{54}$ \\
\hline
\end{tabular}

"Significant diflerence compared to barefoot.

tSignitiant difference compared to thore CAM boot.

Subtalar kinematics for all conditions (barefoot, short CAM boot, and tall CAM boot) are shown in Figure 4 . Barefoot mean maximum and minimum plantarflexion and dorsiflexion were $5.3 \pm 5.6$ degrees and $0.7 \pm 3.2$ degrees, respectively, occurring at $100 \%$ and $9 \%$ stance phase. Short CAM boot mean maximum and minimum plantarflexion and dorsiflexion were $4.1 \pm 5.9$ degrees and $0.7 \pm 2.9$ degrees, respectively, occurring at $91 \%$ and $9 \%$ stance phase. Tall CAM boot mean maximum and minimum plantarflexion were $3.0 \pm 4.7$ degrees and $0.1 \pm$ 4.8 degrees, respectively, occurring at $95 \%$ and $13 \%$ stance phase. Subtalar mean ROM decreased from barefoot to short CAM boot to tall CAM boot from $4.6 \pm 3.9$ degrees to $3.4 \pm 3.8$ degrees to $2.9 \pm 2.6$ degrees (Table 3 ). There was a significant difference between subtalar mean ROM measurements between barefoot and tall CAM boot. 
Table 3. Subtalar Kinematics During Stance Phase.

\begin{tabular}{|l|l|l|l|l|l|}
\hline & $\begin{array}{l}\text { Barefoot, } \\
\text { degrees }\end{array}$ & $\begin{array}{l}\text { Short CAM Boot, } \\
\text { degrees }\end{array}$ & $\begin{array}{l}\text { Tall CAM Boot, } \\
\text { degrees }\end{array}$ \\
\hline & Mean (SD) & Mean (SD) & Mean (SD) \\
\hline Maximum plantarflexion & $5.3(5.6)$ & $4.1(5.9)$ & $3.0(4.7)$ \\
\hline Minimum plantarflexion & $0.7(3.2)$ & $0.7(2.9)$ & $0.1(4.8)$ \\
\hline Range of motion & $4.6(3.9)$ & $3.4(3.8)$ & $2.9(2.6)^{*}$ \\
\hline
\end{tabular}

-Significant difference compared to barefoot.

Table 3. Subtalar Kinematics During Stance Phase.

\begin{tabular}{|c|c|c|c|}
\hline & Barefoot, degrees & Short CAM Boot, degrees & $\mathrm{T}_{2}$ ll CAM Boot, degrees \\
\hline & Mean (SD) & Mean (SD) & Mean (SD) \\
\hline Maximum plantarflexion & $5.3(5.6)$ & $4.1(5.9)$ & $3.0(4.7)$ \\
\hline Minimum plantarflexion & $0.7(3.2)$ & $0.7(2.9)$ & $0.1(4.8)$ \\
\hline Range of motion & $4.6(3.9)$ & $3.4(3.8)$ & $2.9(2.6)^{\circ}$ \\
\hline
\end{tabular}

"Sigificant dillerence compared to barelooe.

\section{Discussion}

The barefoot talocrural sagittal plane kinematics reported in this study are similar in magnitude and trend with those previously reported by our group.., 9 In a 13-subject study published in 2014 , we reported 11.2 degrees of maximum talocrural plantarflexion and -7.5 degrees of maximum dorsiflexion (18.1 degrees of ROM in the sagittal plane). - The currently reported maximum plantar and dorsiflexion angles are 9.2 degrees and -7.5 degrees, respectively (16.7 degrees ROM). Both studies showed barefoot sagittal plane talocrural joint motion in a slightly plantarflexed position at heel strike, with maximum plantarflexion occurring between $9 \%$ and $11 \%$ of stance phase and maximum dorsiflexion occurring between $82 \%$ and $85 \%$ of stance.

Although the barefoot subtalar sagittal plane ROM reported in this study is smaller than previously reported by our group, the trend of the kinematic motion is quite similar. .9 In a previous study of 13 subjects, we reported 4.8 degrees of maximum talocrural plantarflexion and -3.6 degrees of maximum dorsiflexion (8.4 degrees of ROM in the sagittal plane). .9 The currently reported maximum plantar and dorsiflexion angles are 5.3 and 0.7 degrees, respectively (4.6 degrees ROM). Both studies showed the barefoot subtalar in a relatively neutral position at heel strike and then moving toward dorsiflexion, with maximum plantarflexion occurring at the end of the stance phase. Differences between the previously reported and current kinematics likely reflect the effects of small sample studies as a larger database is constructed with this unique kinematic information. Similar differences in ankle kinematics are noted in other human motion analyses studies, with larger population sizes of healthy ambulators. $\underline{10,12,16}$

Comparing the 3 walking conditions (barefoot, short CAM boot, and tall CAM boot) reveals changes in dynamic ranges of joint motion as well as slight offsets (shifts) in the mean value. In the talocrural joint, there was a $52.1 \%$ reduction in sagittal plane talocrural ROM when comparing short CAM boot to barefoot (16.7 degrees barefoot and 8.0 degrees in the short CAM boot), and an $86.8 \%$ reduction when comparing tall CAM boot to barefoot (16.7 degrees barefoot and 2.2 degrees in the tall CAM boot). The difference in ROM between the 2 CAM boot conditions likely occurs because the tall CAM boot has a more proximal tibial contact location than the short CAM boot. Tibial contact location was defined as the most proximal point on the tibia constrained by the CAM boot (Figure 1). This more proximal contact allows for greater control of talocrural motion. In the subtalar joint, there was a $26.1 \%$ reduction in sagittal plane ROM when comparing short CAM boot to barefoot (4.6 degrees barefoot and 3.4 degrees in the short CAM boot), and a $37.0 \%$ reduction when comparing tall CAM boot to barefoot ( 4.6 degrees barefoot and 2.9 degrees in the tall CAM boot). Similar to the talocrural joint, the 
increased distance between the subtalar joint and the tibial contact location in the tall CAM boot is likely the reason for the reduced ROM when compared with the short CAM boot.

A post hoc power analysis of the current study was done for the mean ROM between the barefoot and short CAM boot condition as the short CAM boot had the closest mean ROM value compared to barefoot for both the talocrural and subtalar joints (Tables 2 and $\underline{3}$ ). For the talocrural joint ( $\mu 1=16.7$ degrees, $\mu 2=8.0$ degrees, $\sigma=$ 5.1 degrees), a power of $1.0(\alpha=.05)$ was achieved with the smallest sample size $(n=60$ trials). For the subtalar joint ( $\mu 1=4.6$ degrees, $\mu 2=3.4$ degrees, $\sigma=3.9$ degrees), a power of .39 ( $\alpha=.05$ ) was achieved with the smallest sample size ( $n=60$ trials). In order to achieve a power of .80 for the subtalar joint, 166 trials in each condition (barefoot and short CAM boot) is recommended.

To our knowledge, this is the only gait study comparing talocrural and subtalar barefoot kinematics to short and tall walking boots. In 2006, Zhang et al compared ankle kinematics in shod walking to that in 2 different "shortleg" walking boots and reported no major changes in the peaks or ROMs in the sagittal plane. ${ }^{17}$ Methodological differences, including the lack of fluoroscopic data in the Zhang study, as well as different types of walking boots tested may account for the differences between the results of the Zhang study and the current study.

In conclusion, CAM boot intervention was shown to greatly control sagittal plane talocrural and subtalar joint kinematics compared to barefoot. In addition, tall CAM boot intervention was shown to better control sagittal plane talocrural and subtalar joint kinematics compared to short CAM boot. While these results provide evidence supporting the ability of both tall and short CAM boots to control sagittal plane ankle joint complex motion, further studies should be done with larger populations of both healthy and pathologic feet. The current study used a single fluoroscope with a focused analysis on the sagittal plane only. A biplane fluoroscopic system would be required to report on triaxial kinematics, and our group has recently published the technical details of such a system. $-\frac{3}{A}$ further limitation was the use of ionizing radiation with current levels estimated at 10 $\mu \mathrm{Sv} /$ trial. However, this is well below the United States Nuclear Regulatory Commission (USNRC) whole body annual occupational limits of 5 rems $(50,000 \mu \mathrm{Sv})$.

\section{Declaration of Conflicting Interests}

The author(s) declared the following potential conflicts of interest with respect to the research, authorship, and/or publication of this article: Benjamin D. McHenry, PhD, and Karen M. Kruger, PhD, report grants from US Department of Health and Human Services, National Institute on Disability, Independent Living, and Rehabilitation Research (NIDILRR), during the conduct of the study. ICMJE forms for all authors are available online.

\section{Funding}

The author(s) disclosed receipt of the following financial support for the research, authorship, and/or publication of this article: Medical College of Wisconsin, Department of Orthopaedic Surgery Research Committee; US Department of Health and Human Services, Administration for Community Living, National Institute on Disability, Independent Living, and Rehabilitation Research (90AR5022-02-01; H133P100008).

\section{References}

1. Arndt, A, Westblad, P, Winson, I. Ankle and subtalar kinematics measured with intracortical pins during the stance phase of walking. Foot Ankle Int. 2004;25(5):357-364.

2. Crincoli, MG, Trepman, E. Immobilization with removable walking brace for treatment of chronic foot and ankle pain. Foot Ankle Int. 2001;22(9):725-730.

3. Cross, JA, McHenry, BD, Molthen, R. Biplane fluoroscopy for hindfoot motion analysis during gait: a model-based evaluation. Med Eng Phys. 2017;43:118-123. 
4. DiLiberto, FE, Baumhauer, JF, Wilding, GE. Alterations in plantar pressure with different walking boot designs. Foot Ankle Int. 2007;28(1):55-60.

5. Kadakia, AR, Espinosa, N, Smerek, J. Radiographic comparison of sagittal plane stability between cast and boots. Foot Ankle Int. 2008;29(4):421-426.

6. Keefer, M, King, J, Powell, D. Effects of modified short-leg walkers on ground reaction force characteristics. Clin Biomech. 2008;23(9):1172-1177.

7. Lundgren, $P$, Nester, C, Liu, A. Invasive in vivo measurement of rear-, mid- and forefoot motion during walking. Gait Posture. 2008;28(1):93-100.

8. McHenry, BD, Exten, E, Long, JT, Harris, GF. Sagittal fluoroscopy for the assessment of hindfoot kinematics. J Biomech Eng. 2016;138(3):4032445.

9. McHenry, BD, Exten, EL, Long, J, Law, B, Marks, RM, Harris, G. Sagittal subtalar and talocrural joint assessment with weight-bearing fluoroscopy during barefoot ambulation. Foot Ankle Int. 2015;36(4):430-435.

10. Monaghan, K, Delahunt, E, Caulfield, B. Ankle function during gait in patients with chronic ankle instability compared to controls. Clin Biomech. 2006;21(2):168-174.

11. North, K, Potter, MQ, Kubiak, EN. The effect of partial weight bearing in a walking boot on plantar pressure distribution and center of pressure. Gait Posture. 2012;36(3):646-649.

12. Oberg, T, Karsznia, A, Oberg, K. Basic gait parameters: reference data for normal subjects, 10-79 years of age. J Rehabil Res Dev. 1993;30(2):210.

13. Pollo, FE, Gowling, TL, Jackson, RW. Walking boot design: a gait analysis study. Orthopedics. 1999;22(5):503-507.

14. Raikin, SM, Parks, BG, Noll, KH., Biomechanical evaluation of the ability of casts and braces to immobilize the ankle and hindfoot. Foot Ankle Int. 2001;22(3):214-219.

15. Richards, J, Payne, K, Myatt, D. Do orthotic walkers affect knee and hip function during gait? Prosthet Orthot Int. 2016;40(1):137-141.

16. Steinwender, G, Saraph, V, Scheiber, S. Intrasubject repeatability of gait analysis data in normal and spastic children. Clin Biomech. 2000;15(2):134-139.

17. Zhang, S, Clowers, KG, Powell, D. Ground reaction force and 3D biomechanical characteristics of walking in short-leg walkers. Gait Posture. 2006;24(4):487-492. 